\title{
Aplicação de estudos de QSAR-2D em derivados 5-nitro-2-tiofilidênicos com atividade antimicrobiana frente a Staphylococcus aureus multi- resistente (MRSA)
}

\author{
Andrea Masunari, Leoberto Costa Tavares* \\ Laboratório de Planejamento e Desenvolvimento de Fármacos, Departamento de Tecnologia Bioquímico- \\ Farmacêutica, Faculdade de Ciências Farmacêuticas, Universidade de São Paulo
}

*Correspondência:

L. C. Tavares

Departamento de Tecnologia

Bioquímico-Farmacêutica

Faculdade de Ciências

Farmacêuticas - FCF/USP

Av. Prof. Lineu Prestes n. 580

05505-900 - São Paulo, SP - Brasil

E-mail: leoberto@usp.br
O avanço de estudos de QSAR (Quantitative Structure-Activity Relationships) como método de modificação molecular racionalmente planejada vem se constituindo, nos últimos anos, em alternativa bastante viável para o desenvolvimento de ligantes candidatos potenciais a fármacos. Sendo assim, aliando a excelente aplicabilidade deste método com ofenômeno de multi-resistência bacteriana, realizouse, neste trabalho, o estudo, em duas dimensões, das relações quantitativas entre a estrutura química e a potência antimicrobiana de 5-nitro-2-tiofilideno benzidrazidas substituidas, considerando faixa de hidrofobicidade ótima pré-determinada experimentalmente. A aplicação da Análise de Hansch/Fujita possibilitou a determinação da influência de descritores estruturais de caráter físico-químico sobre a referida atividade, indicando ser a hidrofobicidade a propriedade de maior impacto no desempenho da atividade biológica analisada. Evidenciou-se, a partir das correlações obtidas, o forte potencial de derivados 5-nitro-2-tiofilidênicos como possível alternativa para o desenvolvimento racional, em nível molecular, de novos fármacos com atividade antimicrobiana.
Uniterms

- Staphylococcus aureus

- Multi-resistência

- Nifuroxazida

- QSAR

- Análise de Hansch/Fujita

\section{INTRODUÇÃO}

O emprego da meticilina e outras penicilinas sintéticas como a oxacilina, iniciado em 1959, representou etapa significativa na terapia antiestafilocócica em âmbito mundial. Contudo, a identificação de cepas de Staphylococcus aureus resistentes à meticilina (MRSA - Methicillin-Resistant Staphylococcus aureus) foi registrada em 1962, espalhandose rapidamente pelo mundo nos anos subseqüentes.

A partir da década de 1980, as ocorrências de infecções por MRSA aumentaram consideravelmente em vári- os países, sendo alguns destes detectados na Austrália (surgimento em 1985 e disseminação entre 1994-1997) (Torvaldsen, 1999), na Bélgica (Crowcroft, 1999) e em Manitoba (surgimento em 1993) (Nicolle, 1999). Em 1994, Espanha, França e Itália eram considerados os países com maiores índices de infecção por MRSA (Voss, 1994).

Ainda hoje, se observa número crescente de estudos relacionados à epidemiologia deste microrganismo em centros que comportam pacientes imunocomprometidos (Lizioli et al., 2003), que sofreram intervenção cirúrgica (McDonald et al., 2003), que foram transplantados (Desai et al., 2003) ou 
que são portadores de queimaduras graves (Fuchs et al., 2002), ressaltando a necessidade de desenvolvimento de fármacos que sejam eficazes no tratamento dessas infecções.

Apesar do conceito de que a estrutura química de compostos biologicamente ativos e a sua atividade biológica estão intimamente relacionados, ser consideravelmente antigo (van de Waterbeend, 1992; Tute, 1990), grande impulso em estudos dessa área ocorreu apenas há cerca de quatro décadas, quando Hansch e Fujita (1964) propuseram um método voltado para o estudo de relações quantitativas entre estrutura química e atividade biológica.

A Análise de Hansch/Fujita se aplica em estudos de séries de compostos que apresentam estruturas químicas similares, sendo que, em linhas gerais, tais estruturas diferem entre si por variações de grupos substituintes em posições definidas. Neste modelo há a correlação entre valores de atividade biológica de série de compostos com suas respectivas propriedades físico-químicas, na tentativa de identificar e caracterizar a influência de cada fator físico-químico envolvido no desencadeamento da atividade biológica. Citase que esta correlação pode se dar por regressão linear, regressão linear múltipla ou regressão não-linear (Tavares, 2004; Gaudio, 1996; Kubinyi, 1993).

Apesar de relativamente antiga, a Análise de Hansch/Fujita ainda é amplamente utilizada em trabalhos de química medicinal, já que estudos em duas dimensões (QSAR-2D) são de extrema importância pelo fato de fornecerem subsídios imprescindíveis para a realização de estudos em três (QSAR-3D) (Kubinyi, 1993) ou até quatro dimensões (QSAR-4D) (Hopfinger, 1997). Em outras palavras, pode-se afirmar que estudos realizados em duas dimensões constituem base experimental segura para a posterior aplicação de outros em três ou quatro dimensões. Estes últimos, apesar de constituírem grande avanço na área de química medicinal, apresentam caráter predominantemente teórico, ressaltando, ainda, o fato de que grande parte dos programas disponíveis não é auto-suficiente a ponto de se dispensar dados experimentais.

Apresentam-se como principais vantagens da Análise de Hansch/Fujita a predição da atividade biológica de compostos análogos ainda desconhecidos, a otimização das propriedades de interesse de compostos com perfil farmacológico definido (Tute, 1990) e a modificação da atividade biológica intrínseca (Lien, 1987).

Compostos nitrofurânicos têm sido utilizados desde a década de 1940 para o tratamento de infecções do trato urinário, sendo os primeiros fármacos nitro-heterocíclicos introduzidos na quimioterapia (Venuti, 1995). Número considerável de compostos nitrofurânicos com atividade antibacteriana são aplicados em medicina veterinária e humana, já que estes apresentam amplo espectro de ação e baixa capacidade de indução de resistência bacteriana (Petri Jr., 2001; Dayan, Deguingand, Truzman, 1985; Vozyakova et al., 1978).

Estudos relacionados à atividade antimicrobiana de compostos 5-nitrofurânicos mostraram que a maioria dos derivados ativos apresentava amplo espectro de ação, agindo não apenas sobre bactérias Gram-positivas e Gram-negativas como, algumas vezes também, sobre protozoários (Tavares et al., 1999a, 1999b; El-Obeid, Elnima, Al-Badar, 1985; Avril et al., 1980; Carron et al., 1962). Observa-se, no entanto, que apenas alguns desses derivados são utilizados na prática médica. Isso se deve tanto ao fato da maioria desses compostos apresentar amplo espectro de ação antibacteriana (Petri Jr, 2001; Piscopo et al., 1989; Hirano et al., 1967; Carron et al., 1962; Green, 1948), como também pelo fato de que, embora muitos destes exibam boa atividade, alguns apresentam como fator limitante a alta toxicidade (Foye, Lemke, Willians, 1995; Korolkovas, 1988; Korolkovas, Burckhalter, 1982; Bambury, 1980; Carron et al., 1962). A furilfuramida, por exemplo, que se apresenta mutagênica em testes in vitro e carcinogênica in vivo, foi banida como aditivo alimentar no Japão na década de 1970 (Bryan, 1978). Outros fármacos desta classe, porém, como a nitrofurantoína e a nifuroxazida ainda são utilizados como agentes antibacterianos em medicina humana e veterinária por apresentarem toxicidade tolerável. A nitrofurantoína e o nifuratel são utilizados em infecções do trato genito-urinário causadas principalmente por bactérias Gram-negativas ou protozoários, enquanto que a furazolidona e a nifuroxazida são empregadas no combate a infecções do trato gastrintestinal causadas por bactérias Gram-positivas ou protozoários (Petri Jr, 2001; Foye, Lemke, Willians, 1995; Korolkovas, 1988; Korolkovas, Burckhalter, 1982; Bambury, 1980; Bryan, 1978).

Compostos nitrofurânicos utilizados como antibacterianos apresentam em comum o grupo azometínico $(-\mathrm{CH}=\mathrm{N}-\mathrm{N}<)$ ligado ao carbono 2 do anel 5-nitrofurânico, resultando provavelmente em sinergismo farmacológico entre estas duas subestruturas (Foye, Lemke, Willians, 1995; Korolkovas, 1988). Apresenta-se, na Figura 1, o grupo farmacofórico de compostos nitrofurânicos com atividade antibacteriana.

Como pré-requisito para o desencadeamento da atividade biológica em compostos nitrofurânicos observa-se a bioativação proporcionada pela redução do grupo nitro, que, ao atuar como aceptor de elétrons, resulta na perturbação do fluxo fisiológico de elétrons, inibindo enzimas envolvidas no metabolismo do piruvato (Murray et al., 1996), processo essencial para a produção de energia celular (Tocher, 1997; Ulmar, Mitchard, 1968; Asnis, Gots, 1951; Green, 1948).

Apesar de constituir foco de estudo de muitos grupos de pesquisa, o mecanismo de ação de derivados 5- 


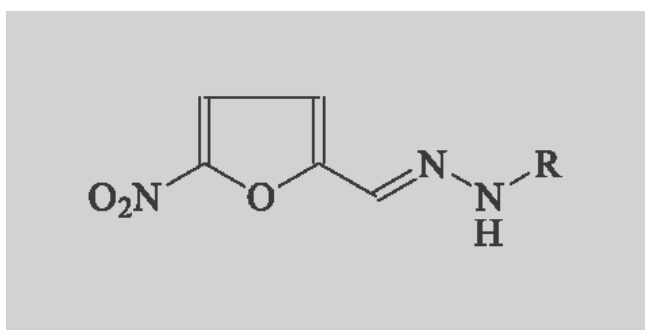

FIGURA 1 - Grupo farmacofórico de compostos nitrofurânicos com atividade antibacteriana.

nitrofurânicos ainda não está totalmente elucidado. Considera-se, atualmente, como mecanismo de ação mais provável de compostos nitrofurânicos aquele proposto para o nifurtimox por Viodé e colaboradores (1999). Apresentam-se, na Figura 2, duas possíveis rotas de redução propostas para a elucidação da bioativação de compostos nitrofurânicos.

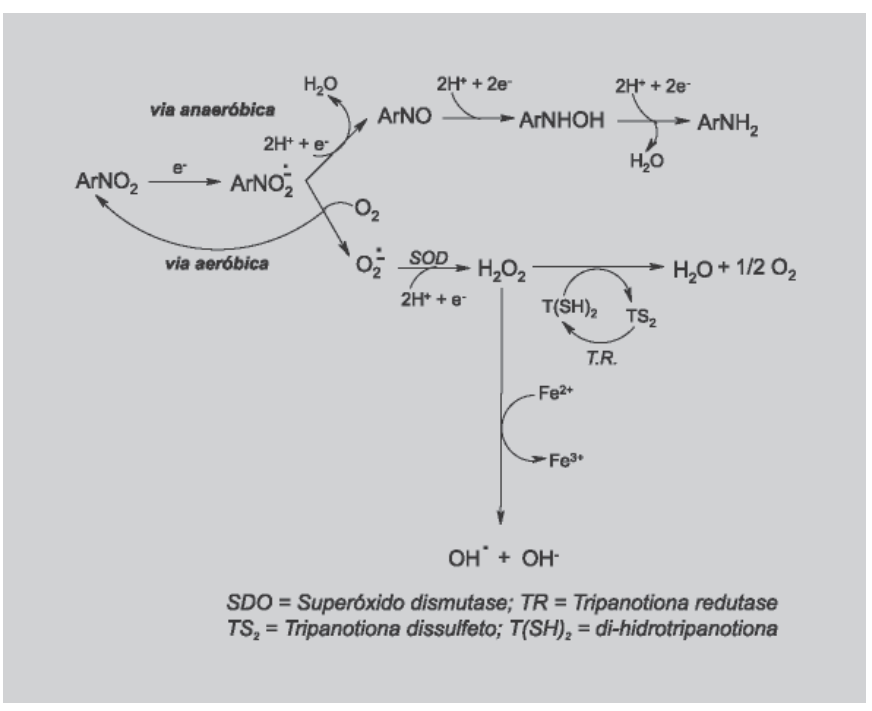

FIGURA 2 - Esquema das vias biorredutivas de compostos nitroaromáticos (Viodé et al., 1999).

$\mathrm{Na}$ rota anaeróbica observa-se que o processo de biorredução do grupo nitro ocasiona acúmulo do radical nitroânion gerado na primeira etapa, sendo que este, em seguida, é transformado no derivado nitroso correspondente. A etapa final deste processo se dá por formação do derivado amino. Já na rota aeróbica, o radical nitroânion formado é inicialmente oxidado a ânions superóxidos (Sreider, Grinblat, Stoppani, 1990), que, sob influência da superóxido desmutase (SOD), geram peróxido de hidrogênio. O acúmulo deste último proporciona a formação de radicais hidroxila, que, ao reagirem com macromoléculas celulares, resultam em danos biológicos como lesão da membrana, inativação enzimática e metagênese (Viodé et al., 1999; Tocher, 1997).

Tavares e colaboradores (Tavares et al., 1999a,b; Tavares, Amaral, 1991) mostraram em estudos envolvendo modificações estruturais planejadas na molécula da nifuroxazida, 5-nitro-2-furfurilideno-4-hidroxibenzidrazida, Figura 3, que substituições da hidroxila fenólica por grupos retiradores de elétrons e do anel furânico por tiofênico favoreceram significativamente a atividade antimicrobiana.

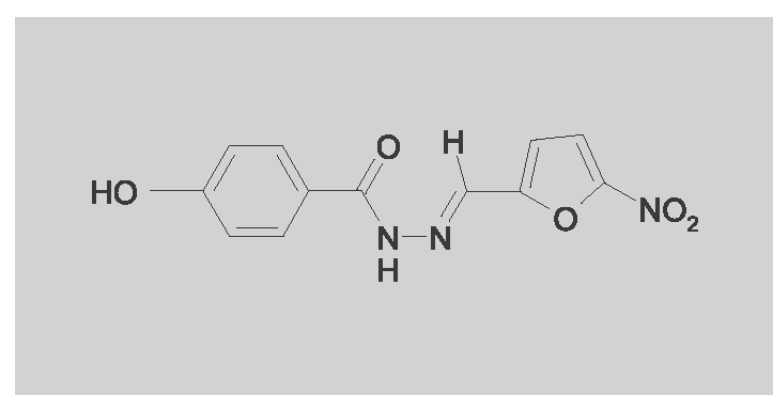

FIGURA 3 - Estrutura química da nifuroxazida.

Estudos comparativos da atividade antimicrobiana de derivados furânicos e tiofênicos com estrutura análoga à nifuroxazida mostraram que para os $p$-acetil derivados, o valor de concentração inibitória mínima do derivado tiofênico mostrou-se cerca de quatro vezes menor que o determinado para o derivado furânico (Tavares et. al., 1999a), sendo constatado para este último potência seis vezes maior que a da nifuroxazida, indicando, portanto, o caráter promissor desta série de compostos para aplicação de estudos de relações estrutura-atividade mais aprofundados.

Assim, com a finalidade de se determinar quantitativamente a influência de descritores estruturais de caráter físico-químico na atividade antimicrobiana de 5-nitro-2-tiofilideno benzidrazidas substituídas, realizou-se, neste estudo, a aplicação de estudos de QSAR-2D, correlacionando a potência de dezoito análogos com as propriedades físico-químicas que regem o desencadeamento de seu desempenho, considerando faixa de hidrofobicidade ótima pré-determinada experimentalmente.

\section{MATERIAL E MÉTODOS}

\section{Compostos em estudo}

Foram avaliadas dezoito 5-nitro-2-tiofilideno benzidrazidas $p$-X-substituídas, em que $\mathrm{X}=\mathrm{H}, \mathrm{Cl}, \mathrm{I}, \mathrm{Br}, n$ $\mathrm{C}_{3} \mathrm{H}_{7}, i-\mathrm{C}_{3} \mathrm{H}_{7}, n-\mathrm{C}_{4} \mathrm{H}_{9}, \mathrm{OC}_{3} \mathrm{H}_{7}, \mathrm{OC}_{4} \mathrm{H}_{9}, \mathrm{NHC}_{4} \mathrm{H}_{9}, \mathrm{CH}=\mathrm{CH}_{2}$, 
$\mathrm{COCH}_{3}, \mathrm{C}_{2} \mathrm{H}_{5}, \mathrm{CH}_{3}, \mathrm{CN}, \mathrm{NO}_{2}, \mathrm{SO}_{2} \mathrm{NH}_{2}$. Foi incluído ainda no estudo o derivado $m, p$ - $\mathrm{Cl}_{2}$ dissubstituído. Todos os compostos foram previamente sintetizados e identificados pelo grupo de pesquisa do Laboratório de Planejamento e Desenvolvimento de Fármacos - FBT/FCF-USP (Masunari et al., 2003; Rezende et al., 2002; Furlanetto, Santos, Tavares, 2001), utilizando-se como metodologias de confirmação da estrutura química dos análogos planejados, análises espectrométricas de ressonância magnética nuclear de hidrogênio $\left(\mathrm{RMN}-{ }^{1} \mathrm{H}\right)$ e de carbono $13\left(\mathrm{RMN}-{ }^{13} \mathrm{C}\right)$ e espectrometria de absorção na região do Infravermelho (IV). A determinação da faixa de fusão e a análise elementar foram utilizadas como critério do grau de pureza dos compostos obtidos.

\section{Ensaio microbiológico}

A avaliação da atividade antimicrobiana das 5-nitro2-tiofilideno benzidrazidas substituídas frente às cepas ATCC 25923 e multi-resistente de Staphylococcus aureus (cepa 3SP/R33) (Oliveira et al., 2001) foi realizada por determinação da concentração inibitória mínima, MIC, que envolveu basicamente duas fases. Na primeira etapa, fase I, determinou-se de forma quantitativa a atividade antimicrobiana dos compostos obtidos, utilizando método clássico de macrodiluição sucessiva em tubos (Tavares, 1993; Collins, 1995). Na segunda etapa, fase II, utilizouse metodologia adaptada por Tavares e colaboradores (Tavares, Penna, Amaral, 1997) com o propósito de intensificar a sensibilidade do ensaio.

Na preparação do inóculo, procedeu-se à lavagem das colônias de três tubos com culturas de 24 horas de Staphylococcus aureus em PCA (Plate Count Agar) inclinado, com porções equivalentes a 5,0 mL de solução salina a $0,85 \%$ para cada tubo. Transferiram-se as suspensões obtidas para frasco contendo pérolas de vidro e barra magnética. Manteve-se a suspensão sob agitação constante por 30 minutos para homogeneização do meio. Transferiu-se $1,0 \mathrm{~mL}$ desta suspensão para frasco contendo $99,0 \mathrm{~mL}$ de solução salina, pérolas de vidro e barra magnética, obtendose suspensão de $1,0 \times 10^{-2} \mathrm{UFC} \mathrm{mL}^{-1}$ (unidades formadoras de colônia por $\mathrm{mL}$ ) em relação à suspensão inicial. Manteve-se esta segunda suspensão sob agitação constante por 15 minutos. Repetiu-se este mesmo procedimento para a suspensão seguinte, obtendo-se concentração equivalente a $1,0 \times 10^{-4} \mathrm{UFC} \mathrm{mL}^{-1}$. Por fim, transferiu-se $1,0 \mathrm{~mL}$ da suspensão anterior de $1,0 \times 10^{-4} \mathrm{UFC} \mathrm{mL}^{-1}$ para frasco contendo 99,0 mL de TSB (Tryptic Soy Broth, Merck), pérolas de vidro e barra magnética, gerando inóculo de 0,5 na escala Mc Farland, utilizado na realização do ensaio.

Para cada análogo a ser testado, prepararam-se $10,0 \mathrm{~mL}$ de solução do antimicrobiano com concentração equivalente a $1,8 \times 10^{-3} \mathrm{Mem}$ DMSO (dimetilsulfóxido), para compostos mais hidrossolúveis, e, alternativamente, em DMSO e álcool etílico (co-solvente), na proporção de 1:1 (v/v), para os compostos mais lipossolúveis. Foram transferidos 5,0 mL desta solução para frasco contendo $5,0 \mathrm{~mL}$ de TSB, de forma a originar solução de concentração equivalente a $0,9 \times 10^{-3} M$, posteriormente empregada na execução do ensaio.

Ressalta-se, ainda, que todos os compostos testados apresentaram valores de concentração inibitória mínima em região com concentração de DMSO muito abaixo de seu limite crítico $(12,5 \%)$, permitindo atribuir a inibição do crescimento microbiano apenas à atividade antibacteriana inerente aos compostos em estudo.

\section{Estudo de QSAR: Análise de Hansch/Fujita}

Os estudos de QSAR foram realizados por aplicação da Análise de Hansch/Fujita com a finalidade de avaliar, de forma quantitativa, a influência da hidrofobicidade e dos efeitos eletrônico e estéreo sobre a atividade antimicrobiana. Para tal, utilizaram-se os valores de concentração inibitória mínima frente à cepa 3SP/R33 de Staphylococcus aureus, expressos em potência (Log 1/MIC) em função de descritores estruturais que expressam a hidrofobicidade e as propriedades eletrônica e estereoquímica.

Os descritores estruturais utilizados neste estudo para representar a hidrofobicidade foram o logaritmo do coeficiente de partição calculado, $C \log \mathrm{P}(C \log P$ Program versão 4.0, Biobyte Co.) e a constante de hidrofobicidade, $\pi$ (Kubinyi, 1993; Hansch, Fujita, 1964), proposta por Hansch.

Como descritores para a avaliação da distribuição eletrônica foram utilizadas a constante de grupo de Hammett, $\sigma$ (1937), e as constantes $\mathfrak{I}$ e $\mathfrak{R}$ de Swain e Lupton (Swain, Lupton, 1969).

$\mathrm{O}$ efeito estéreo foi representado pelos parâmetros de Verloop, L, B1, B2, B3, B4 e B5 (Verloop, 1987), ressaltando, ainda, a introdução da refratividade molar (RM) (Kubinyi, 1993), um descritor estrutural de caráter misto.

Aplicou-se o método de stepwise (análise por etapas), sendo as correlações efetuadas por aplicação do Billin Program, versão 1998, desenvolvido e disponibilizado pelo Prof. Dr. Hugo Kubinyi (BASF). Efetuou-se a análise dos resultados obtidos pela interpretação das equações (modelos linear, parabólico e bilinear) e gráficos de dispersão.

Assim, a potência, considerada a variável dependente, foi correlacionada com os descritores estruturais relacionados a cada propriedade físico-química analisada, variáveis independentes, originando equações cuja interpretação foi feita através da análise dos parâmetros estatísti- 
cos depreendidos da regressão.

A avaliação dos modelos foi realizada por procedimentos comuns. A saber: verificação do grau de ajuste do modelo, que envolve o cálculo do coeficiente de correlação (r) e do desvio-padrão (s); verificação do grau de significância do modelo pelo cálculo do parâmetro de significância de Fisher ou teste F (95\% de confiança); verificação do grau de previsibilidade do modelo pelo teste de validação cruzada com cálculo subseqüente do coeficiente de correlação $\left(Q^{2}\right)$ e do desvio padrão das previsões $\left(\mathrm{s}_{\text {PRESS }}\right)$ (Kubinyi, 1993).

\section{RESULTADOS E DISCUSSÃO}

\section{Estudos das relações estrutura-atividade (SAR)}

Com a finalidade de comprovar a influência da hidrofobicidade na atividade antimicrobiana de derivados 5nitrofurânicos, Tavares e colaboradores (1999b) associaram o esquema operacional de Topliss (1972) ao diagrama de Craig (1971)em série de análogos à nifuroxazida com a finalidade de avaliar a influência de grupos substituintes na referida atividade. Utilizou-se o diagrama de Craig com a finalidade de ampliar o número de análogos estudados, de forma a estabelecer modelos matemáticos que explicassem quantitativamente a influência da hidrofobicidade na atividade dos análogos em questão. Comprovou-se, em concordância com os estudos previamente realizados pelo mesmo grupo (Tavares et al., 1999a; Tavares, 1993), que a hidrofobicidade constitui-se em propriedade físico-química fundamental na determinação da atividade antimicrobiana dos derivados 5-nitro-2-furfurilidênicos.

Com base nestas informações, efetuou-se, neste trabalho, a substituição do anel furânico por tiofênico, de forma a proporcionar maior hidrofobicidade à série de análogos em estudo, ao mesmo tempo em que se aplicou, numa primeira etapa, o esquema operacional de Topliss, que visa, essencialmente, à obtenção de compostos com atividade biológica otimizada.

Seguindo o esquema proposto por Topliss (Topliss, 1972) constatou-se, por meio da determinação da concentração inibitória mínima dos compostos sintetizados frente à cepa multi-resistente (3SP/R33) de Staphylococcus aureus, que o ramo da árvore decisória a ser seguido estaria relacionado ao aumento da hidrofobicidade dos compostos (Figura 4).

A aplicação do esquema operacional de Topliss na presente série indicou, em concordância com os estudos

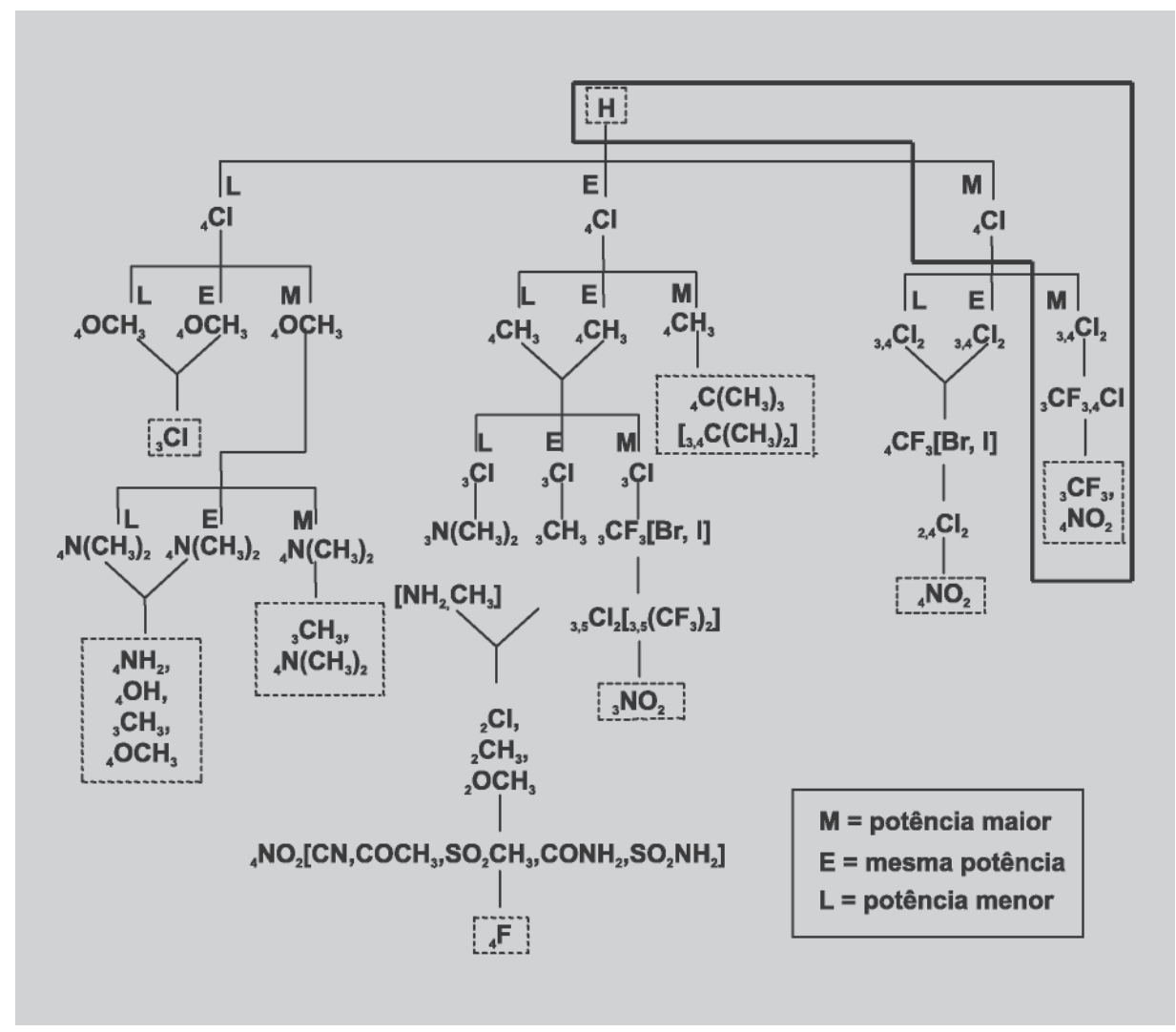

FIGURA 4 - Ramo da árvore decisória proposta por Topliss, que apresentou acréscimo na atividade antimicrobiana da série de análogos estudados. 
anteriormente realizados por este grupo de pesquisa, que o aumento da potência antimicrobiana estaria diretamente relacionado ao aumento da hidrofobicidade dos análogos por influência dos grupos substituintes.

Com base nestes resultados, selecionaram-se mais dez grupos substituintes de acordo com o Diagrama de Craig (1971), considerando como intervalo de hidrofobicidade a faixa de valores de $\pi$ entre 0,70 e 1,55, faixa identificada como ótima pela aplicação do diagrama de Topliss. Os valores de $\pi$ considerados como limite foram os correspondentes aos grupos $p-\mathrm{Cl}$ e $p-\mathrm{Cl}, m-\mathrm{CF}_{3}$, respectivamente.

A escolha desta faixa de valores de $\pi$ nada mais é do que o resultado prático de estudos relacionados à determinação da atividade antimicrobiana de 5-nitro-2-tiofilideno benzidrazidas substituídas realizados em nosso laboratório, em que os compostos que se mostraram mais ativos apresentam valores, ou somatória de $\pi$, equivalentes a 0,86 e 1,42.

Observa-se que a contribuição hidrofóbica inerente a estes dois grupos substituintes, expressa por seus valores de $\pi$, situa-se dentro da faixa por nós estabelecida, de forma a englobar os dois compostos mais potentes obtidos em diferentes trabalhos desenvolvidos em nosso grupo de pesquisa.

A seleção de grupos substituintes com constante de hidrofobicidade fora dos limites estabelecidos pelo estudo como $p-\mathrm{COCH}_{3}(\pi=-0,55), p-\mathrm{CH}_{3}(\pi=0,56), p-\mathrm{CN}$ $(\pi=-0,57), p-\mathrm{NO}_{2}(\pi=-0,28)$ e $p-\mathrm{SO}_{2} \mathrm{NH}_{2}(\pi=-1,82)$ foi efetuada para considerar maior dispersão no diagrama de Craig e evitar intercorrelação entre parâmetros.

Foi possível, assim, identificar experimentalmente, pela aplicação do diagrama operacional de Topliss, um valor aproximado da contribuição hidrofóbica inerente aos grupos substituintes selecionados $(\mathrm{p} \leq 0,70 \pm$ $0,1)$, definido como ótimo. Desta forma, valores de $\pi$ muito acima ou muito abaixo de 0,70 resultaram em considerável decréscimo da potência antimicrobiana, com exceção do composto $p$-acetil substituído, que exibiu comportamento flagrantemente distinto do observado para toda a série em estudo.

Apresenta-se, no item a seguir, a análise quantitativa da série de análogos 5-nitro-2-tiofilidênicos com a atividade antimicrobiana estudados neste trabalho.

\section{Estudos das relações quantitativas estrutura-atividade em duas dimensões - QSAR-2D}

Foram obtidas correlações que associaram a potência antimicrobiana a uma, duas e três variáveis independentes distintas representadas por descritores estruturais de caráter hidrofóbico, eletrônico e estéreo.
Apresentam-se, nas Tabelas I e II, os valores dos parâmetros biológicos e dos descritores estruturais de caráter físico-químico referentes a cada composto analisado neste estudo.

TABELA I - Potência antimicrobiana das 5-nitro-2tiofilideno benzidrazidas substituídas

\begin{tabular}{lccc}
\hline $\mathbf{R}_{1}$ & $\mathbf{R}_{2}$ & MIC(mM) & $\begin{array}{c}\text { Potência } \\
\text { Log } \mathbf{1} / \mathbf{M I C}\end{array}$ \\
\hline $\mathrm{H}$ & $\mathrm{H}$ & 16,67 & 4,78 \\
$\mathrm{Cl}$ & $\mathrm{H}$ & 5,52 & 5,26 \\
$\mathrm{Cl}$ & $\mathrm{Cl}$ & 2,21 & 5,66 \\
$\mathrm{I}$ & $\mathrm{H}$ & 14,01 & 4,85 \\
$\mathrm{Br}$ & $\mathrm{H}$ & 2,12 & 5,67 \\
$n-\mathrm{C}_{3} \mathrm{H}_{7}$ & $\mathrm{H}$ & 36,55 & 4,44 \\
$i-\mathrm{C}_{3} \mathrm{H}_{7}$ & $\mathrm{H}$ & 28,55 & 4,54 \\
$\mathrm{OC}_{3} \mathrm{H}_{7}$ & $\mathrm{H}$ & 24,29 & 4,61 \\
$\mathrm{OC}_{4} \mathrm{H}_{9}$ & $\mathrm{H}$ & 35,41 & 4,45 \\
$\mathrm{NHC}_{4} \mathrm{H}_{9}$ & $\mathrm{H}$ & 22,37 & 4,65 \\
$\mathrm{CH} \mathrm{CH}_{2}$ & $\mathrm{H}$ & 15,66 & 4,80 \\
$n-\mathrm{C}_{4} \mathrm{H}_{9}$ & $\mathrm{H}$ & 40,74 & 4,39 \\
$\mathrm{C}_{2} \mathrm{H}_{5}$ & $\mathrm{H}$ & 22,25 & 4,65 \\
$\mathrm{COCH}_{3}$ & $\mathrm{H}$ & 0,44 & 6,36 \\
$\mathrm{CH}_{3}$ & $\mathrm{H}$ & 13,49 & 4,87 \\
$\mathrm{CN}^{*}$ & $\mathrm{H}$ & 7,02 & 5,15 \\
$\mathrm{NO}_{2} *$ & $\mathrm{H}$ & 5,06 & 5,29 \\
$\mathrm{SO}_{2} \mathrm{NH}_{2} * *$ & $\mathrm{H}$ & 31,75 & 4,50 \\
\hline
\end{tabular}

* Sintetizados e avaliados por Rezende e colaboradores (2002);**Sintetizado e avaliado por Furlanetto e colaboradores (2001)

As melhores correlações foram obtidas por aplicação dos modelos parabólico e bilinear e quando associaram-se dois descritores estruturais em cada equação. A saber: $\mathrm{Clog} \mathrm{P}$ e $\sigma, \mathrm{Clog} \mathrm{P}$ e $\mathfrak{I}$.

$$
\begin{gathered}
\log 1 / \mathrm{MIC}=-0,294( \pm 0,094) \mathrm{ClogP}^{2}+1,540( \pm 0,50) \\
\mathrm{Clog}+0,598( \pm 0,27) \mathrm{s}+2,919( \pm 0,66) \\
\quad \log \mathrm{P}_{\text {ótimo }}=2,62 \\
(\mathrm{n}=15 ; \mathrm{r}=0,942 ; \mathrm{s}=0,111 ; \mathrm{F}=29,050 ; \\
\left.Q^{2}=0,822 ; \mathrm{s}_{\mathrm{PRESS}}=0,140\right) \\
\text { Equação 1 }
\end{gathered}
$$

$\log 1 / \mathrm{MIC}=0,813( \pm 0,29) \mathrm{C} \log \mathrm{P}-1,454( \pm 0,48) \log$ $\left(ß .10^{\mathrm{Clog} P}+1\right)+0,608( \pm 0,28) \mathrm{s}+3,364( \pm 0,56)$ $\log \beta=-2,499 \mathrm{Clog} \mathrm{P}_{\text {ótimo }}=2,60$ $(\mathrm{n}=15 ; \mathrm{r}=0,945 ; \mathrm{s}=0,113 ; \mathrm{F}=21,069)$ Equação 2 
$\log 1 / \mathrm{MIC}=-0,296( \pm 0,10) \mathrm{Clog} \mathrm{P}^{2}+1,448( \pm 0,52)$

$\mathrm{C} \log \mathrm{P}+0,707( \pm 0,35) \mathfrak{I}+3,077( \pm 0,67)$

$$
\mathrm{Clog} \mathrm{P}_{\text {ótimo }}=2,44
$$

( $\mathrm{n}=15 ; \mathrm{r}=0,934 ; \mathrm{s}=0,119 ; \mathrm{F}=25,027$;

$$
\left.Q^{2}=0,632 ; \mathrm{s}_{\text {PRESS }}=0,201\right)
$$

Equação 3

$\log 1 / \mathrm{MIC}=1,024( \pm 0,34) \mathrm{C} \log \mathrm{P}-1,602( \pm 0,49) \log$

$$
\begin{gathered}
\left(ß .10^{\operatorname{Clog} P}+1\right)+0,717( \pm 0,33) \mathfrak{I} \\
+3,201( \pm 0,58) \\
\log \beta=-2,086 \text { ClogP }=2,33 \\
(\mathrm{n}=15 ; \mathrm{r}=0,950 ; \mathrm{s}=0,109 ; \mathrm{F}=23,150) \\
\text { Equação } 4
\end{gathered}
$$

Observou-se que os modelos parabólico e bilinear se adequaram à descrição da influência de propriedades físico-químicas sobre a potência antimicrobiana, ressaltando, ainda, que a exclusão dos compostos $p$-acetil, $p$-bromo e $m, p-\mathrm{Cl}_{2}$ substituídos resultou em melhor ajuste das equa- ções geradas aos dados obtidos experimentalmente, evidenciando, desta maneira, comportamentos distintos destes compostos em relação aos demais componentes da série analisada.

No caso dos compostos $p$-acetil ( $\mathrm{MIC}=0,14 \mathrm{mg} / \mathrm{mL}$ ) e $p$-bromo (MIC $=0,75 \mathrm{mg} / \mathrm{mL}$ ) substituídos foi observado comportamento diferenciado de potência antimicrobiana, permitindo-nos classificá-los como super ativos.

Ainda não é possível afirmar, com segurança, quais interações poderiam estar envolvidas ou quais seriam as principais propriedades físico-químicas responsáveis por tão pronunciada atividade. A exclusão do único composto dissubstituído da série, o derivado $m, p-\mathrm{Cl}_{2}$ substituído, resultando em melhor ajuste das equações aos dados ob-

\begin{tabular}{|c|c|c|c|c|c|c|c|c|c|c|c|c|}
\hline $\mathrm{R}_{1}$ & $\mathrm{R}_{2}$ & $\pi^{(\mathrm{a})}$ & $\mathrm{Clog} \mathrm{P}^{(\mathrm{d})(\mathrm{III})}$ & $\mathrm{MR}^{\text {(a) }}$ & $\sigma^{(\mathrm{a})}$ & $\mathfrak{I}^{(\mathrm{b})}$ & $\Re^{\text {(b) }}$ & $\mathrm{L}^{\text {(a) }}$ & $\mathrm{B}_{1}{ }^{(\mathrm{a})}$ & $\mathrm{B}_{2}^{(\mathrm{a})}$ & $\mathrm{B}_{3}{ }^{(\mathrm{a})}$ & $\mathrm{B}_{4}{ }^{(\mathrm{a})}$ \\
\hline $\mathrm{H}$ & $\mathrm{H}$ & 0,00 & 2,10 & 0,103 & 0,00 & 0,00 & 0,00 & 2,06 & 1,00 & 1,00 & 1,00 & 1,00 \\
\hline $\mathrm{Cl}$ & $\mathrm{H}$ & 0,71 & 2,91 & 0,603 & 0,23 & 0,42 & $-0,19$ & 3,52 & 1,80 & 1,80 & 1,80 & 1,80 \\
\hline $\mathrm{Cl}$ & $\mathrm{Cl}$ & 1,42 & 3,53 & — (II) & 0,60 & — $^{\text {(II) }}$ & — (II) & — (II) & — $^{\text {(II) }}$ & — $^{\text {(II) }}$ & — (II) & — (II) \\
\hline I & $\mathrm{H}$ & 1,12 & 3,32 & 1,394 & 0,18 & 0,42 & $-0,24$ & 4,23 & 2,15 & 2,15 & 2,15 & 2,15 \\
\hline $\mathrm{Br}$ & $\mathrm{H}$ & 0,86 & 3,06 & 0,888 & 0,23 & 0,45 & $-0,22$ & 3,83 & 1,95 & 1,95 & 1,95 & 1,95 \\
\hline $\mathrm{n}-\mathrm{C}_{3} \mathrm{H}_{7}$ & $\mathrm{H}$ & 1,55 & 3,54 & 1,496 & $-0,13$ & 0,01 & $-0,14$ & 5,05 & 1,52 & 3,49 & 1,90 & 1,90 \\
\hline$i-\mathrm{C}_{3} \mathrm{H}_{7}$ & $\mathrm{H}$ & $1,53^{(\mathrm{c})}$ & 3,56 & 1,500 & $-0,15$ & 0,04 & $-0,19$ & - $^{\text {(I) }}$ & - $^{\text {(I) }}$ & - $^{\text {(I) }}$ & - $^{\text {(I) }}$ & - $^{\text {(I) }}$ \\
\hline $\mathrm{OC}_{3} \mathrm{H}_{7}$ & $\mathrm{H}$ & $1,05^{(b)}$ & 3,46 & 1,706 & $-0,25$ & $0,26^{\text {(c) }}$ & $-0,51^{(\mathrm{c})}$ & 6,05 & 1,35 & 4,30 & 1,90 & 1,90 \\
\hline $\mathrm{OC}_{4}^{3} \mathrm{H}_{9}^{\prime}$ & $\mathrm{H}$ & 1,55 & 3,65 & 2,166 & $-0,32$ & 0,29 & $-0,61$ & 6,99 & 1,35 & 4,79 & 1,90 & 1,90 \\
\hline $\mathrm{NHC}_{4} \mathrm{H}_{9}$ & $\mathrm{H}$ & 1,16 & 3,26 & 2,426 & $-0,51$ & $-0,04$ & $-0,57$ & 7,01 & 1,50 & 4,97 & 1,90 & 1,90 \\
\hline $\mathrm{CH}=\mathrm{CH}_{2}$ & $\mathrm{H}$ & 0,82 & 2,98 & 1,099 & $-0,02$ & 0,13 & $-0,17$ & 4,29 & 1,60 & 1,60 & 2,00 & 3,09 \\
\hline$n-\mathrm{C}_{4} \mathrm{H}_{9}$ & $\mathrm{H}$ & 2,13 & 3,95 & 1,969 & $-0,16$ & $-0,01$ & $-0,15$ & 6,17 & 1,52 & 4,42 & 1,90 & 1,90 \\
\hline $\mathrm{C}_{2} \mathrm{H}_{5}$ & $\mathrm{H}$ & 1,02 & 3,10 & 1,030 & $-0,15$ & 0,00 & $-0,15$ & 4,11 & 1,52 & 2,97 & 1,90 & 1,90 \\
\hline $\mathrm{COCH}_{3}$ & $\mathrm{H}$ & $-0,55$ & 1,88 & 1,118 & 0,50 & 0,33 & 0,17 & 4,06 & 1,90 & 1,90 & 2,36 & 2,93 \\
\hline $\mathrm{CH}_{3}$ & $\mathrm{H}$ & 0,56 & 2,60 & 0,565 & $-0,17$ & 0,01 & $-0,18$ & 3,00 & 1,52 & 2,04 & 1,90 & 1,90 \\
\hline $\mathrm{CN}^{3} *$ & $\mathrm{H}$ & $-0,57$ & 1,76 & 0,633 & 0,66 & 0,51 & 0,15 & 4,23 & 1,60 & 1,60 & 1,60 & 1,60 \\
\hline $\mathrm{NO}_{2} *$ & $\mathrm{H}$ & $-0,28$ & 2,05 & 0,736 & 0,78 & 0,65 & 0,13 & 3,49 & 1,70 & 1,70 & 2,44 & 2,44 \\
\hline $\mathrm{SO}_{2} \mathrm{NH}_{2} * *$ & $\mathrm{H}$ & $-1,82$ & 0,98 & 1,228 & 0,60 & $0,49^{\text {(c) }}$ & $0,11^{\text {(c) }}$ & 3,82 & 2,11 & 3,07 & 2,67 & 2,67 \\
\hline
\end{tabular}
tidos, pode ser explicada pelo fato da excelente atividade $(\mathrm{MIC}=0,76 \mathrm{mg} / \mathrm{mL})$ exibida pelo análogo em questão ser condicionada provavelmente pela influência de propriedades relacionadas ao volume dos grupos substituintes en-

TABELA II - Descritores estruturais relacionados aos efeitos hidrofóbico, eletrônico e ao volume de grupos substituintes utilizados na análise de QSAR da série de derivados do 5-nitro-2-tiofilideno

Nota 1: (a) Kubinyi, H. QSAR: Hansch Analysis and Related Approaches. New York: VCR, 1993; (b) Hansch, C., Leo, A. Exploring QSAR: Fundamentals and Applications in Chemistry and Biology. Washington: American Chemical Society, 1995; (c) Hansch, C., Leo, A., Hoekman, D. Exploring QSAR: Hydrophobic, Electronic and Steric Constants. Washington: American Chemical Society, 1995; (d): Calculados pelo ClogP Program, versão 4.0

Nota 2: *Sintetizados e avaliados por Rezende e colaboradores (2002); **Sintetizado e avaliado por Furlanetto e colaboradores (2001)

Nota 3: (I): Valor não disponível em literatura; (II): Valor não disponível em literatura para derivados dissubstituídos; (III): Valores referentes à estrutura molecular de 5-nitro-2-tiofilideno benzidrazidas substituídas 
volvidos, sendo importante ressaltar que o efeito estéreo, apesar de ser praticamente indissociável de propriedades de natureza eletrônica e hidrofóbica, é pouco avaliado na análise clássica de QSAR.

O modelo bilinear apresenta termos probabilísticos que descrevem o deslocamento de compostos químicos no sistema biológico considerando seu caráter multicompartimentar, em que a potência antimicrobiana é altamente dependente da hidrofobicidade. A aplicação deste modelo na correlação entre potência antimicrobiana e os descritores estruturais ClogP, $\mathfrak{I}$ e $\sigma$ resultou em equações que expressam convenientemente a dependência não-linear da potência antimicrobiana em relação à hidrofobicidade (Equações 2 e 4). Assim, dois dados experimentais de extrema importância, que podem ser extraídos das equações 2 e 4 e aplicados em outros estudos de QSAR devido ao seu caráter preditivo, são o coeficiente de partição ótimo, $C_{\log } \mathrm{P}_{\text {ótimo }}$, e o $\log \beta$, sendo o último uma constante de ajuste, que expressa a relação entre volumes das fases orgânica e aquosa, que pode ser aplicada na determinação experimental do coeficiente de partição de ligantes pertencentes a esta série (Kubinyi, 1993).

Correlações com descritores estruturais relacionados com o perfil tridimensional dos substituintes estudados, propostos por Verloop (1987), também foram analisadas, mas resultaram, entretanto, em equações estatisticamente pouco significativas. É importante citar, ainda, que entre as correlações obtidas com os parâmetros de Sterimol, as que envolveram o descritor $\mathrm{B}_{1}$ apresentaram certa tendência de correlação, sendo os resultados obtidos para este descritor de caráter estereoquímico, ferramenta útil no direcionamento de estudos das relações quantitativas estrutura-atividade em três dimensões.

Foram realizadas, também, correlações considerando três descritores estruturais, mas as equações geradas mostraram-se, em sua maioria, não-significativas, impossibilitando a seleção de modelos que quantificassem, com boa margem de segurança estatística, a influência simultânea de três parâmetros na potência antimicrobiana.

Acredita-se que a importante influência da hidrofobicidade, denotada pelo descritor $\mathrm{Clog} \mathrm{P}$, sobre a potência antimicrobiana, estaria primordialmente relacionada à necessidade de um balanço lipofílico-hidrofílico adequado nos derivados tiofilidênicos, a fim de possibilitar a ocorrência de processos de difusão através de membranas biológicas para posterior desencadeamento da referida atividade.

Além da contribuição positiva notória do coeficiente de partição sobre a potência antimicrobiana, outra observação importante está relacionada a interpretações complementares que podem ser extraídas das equações que envolvem os descritores $\sigma$ e $\mathfrak{I}$ na determinação da potência antimicrobiana (Equações 1, 2, 3 e 4).
O descritor $\sigma$ mede a influência do efeito polar, somatória dos efeitos indutivo e de ressonância, na distribuição eletrônica do ligante como um todo, propiciando a interação do mesmo com o receptor por intermédio das duas propriedades citadas. O descritor $\mathfrak{I}$, entretanto, considera apenas o efeito indutivo e estaria relacionado aos campos moleculares gerados ao redor dos grupos substituintes ligados ao anel benzênico, sugerindo provável interação eletrostática com o receptor.

A exploração quantitativa da influência dos efeitos de caráter eletrônico sobre a potência antimicrobiana dos derivados 5-nitro-2-tiofilidênicos, realizada neste trabalho, complementa os resultados de outros estudos em que se considerou a análise quantitativa da influência da hidrofobicidade sobre a potência antimicrobiana de compostos com estrutura análoga à nifuroxazida (Tavares, 2003; Masunari, Tavares, 2002; Masunari, Rezende, Tavares, 2001; Tavares et al., 1999; Tavares, Penna, Amaral, 1997; Tavares, Penna, Amaral, 1991), subsidiando fortemente as avaliações e predições relacionadas à série de derivados 5nitro-2-tiofilidênicos com atividade antimicrobiana.

\section{CONCLUSÕES}

Os estudos de QSAR-2D demonstraram que a potência antimicrobiana das 5-nitro-2-tiofilideno benzidrazidas substituídas sofre influência significativa de duas propriedades físico-químicas, que são a hidrofobicidade e a distribuição eletrônica. A influência positiva da hidrofobicidade sugere a importância de um balanço lipofílico-hidrofílico adequado nos ligantes a fim de possibilitar sua passagem por membranas biológicas, enquanto que a relevância dos descritores estruturais $\sigma$ e $\mathfrak{I}$ na determinação da atividade antimicrobiana sinalizam que a distribuição eletrônica influencia fortemente o aumento da potência antimicrobiana dos análogos considerando tanto a influência dos efeitos indutivo e de ressonância na estrutura química do ligante, como também os campos moleculares gerados em torno de grupos substituintes, resultando em possível interação dos mesmos com área específica do sítio receptor.

Por aplicação da Análise de Hansch/Fujita foram obtidos modelos estatisticamente significativos que auxiliaram na descrição da influência das propriedades eletrônicas e de hidrofobicidade sobre a potência antimicrobiana, sendo os resultados obtidos neste estudo um forte indício do grande potencial da série 5-nitro-2tiofilidênica para o planejamento de ligantes candidatos a fármacos antimicrobianos, voltados para o tratamento de infecções causadas por Staphylococcus aureus com caráter de multi-resistência. 


\section{ABSTRACT}

\section{D QSAR studies of 5-nitro-2-thiophylidene derivatives with antimicrobial activity against multidrug-resistant Staphylococcus aureus (MRSA)}

With the constant advance of QSAR (Quantitative Structure-Activity Relationships) studies as molecular modification methodology, a frequent application of this procedure in many science areas was observed. Besides, the rational ligand development for many diseases has been growing in recent years. Thus, in order to ally these medicinal chemistry advances with the necessity to combat the high incidence of antibioticresistant microorganisms, the purpose of this study was the 2D-QSAR study of $\mathrm{p}$-substituted 5-nitro-2-thiophylidene derivatives with antimicrobial activity against Staphylococcus aureus, considering hydrophobicity range experimentally determined. The statistical significant correlations obtained by Hansch/ Fujita Analysis showed significant influence of hydrophobicity on antimicrobial activity, prove by reasoning the potential of synthesized compounds as a good alternative to the rational development, on molecular level, of selective drugs used to the treatment of infections caused by Staphylococcus aureus multidrug-resistant strains.

UNITERMS: Staphylococcus aureus. Multidrug resistance. Nifuroxazide. QSAR. Hansch/Fujita Analysis.

\section{AGRADECIMENTOS}

Agradecemos à CAPES e à FAPESP pelo suporte financeiro para o desenvolvimento deste trabalho. Agradecemos à Profa. Dra. Elsa Masae Mamizuka, coordenadora do LRNFSA do FBC/FCF/USP, por ter gentilmente cedido a cepa $3 \mathrm{SP} / \mathrm{R} 33$ de $S$. aureus e todo o suporte técnico-científico para a realização dos ensaios. Agradecemos, também, à Profa. Dra. Thereza Christina Vessoni-Penna coordenadora do LCIF do FBT/FCF/USP por ter cedido a cepa ATCC 25923 de $S$. aureus e o suporte técnico-científico para a realização dos ensaios.

\section{REFERÊNCIAS BIBLIOGRÁFICAS}

ASNIS, R.E.; GOTS, J.S. Studies on the action of nitrofurans on bacterial enzime sistems. I. The inhibition of bacterial respiration by furacin. Arch. Biochem., New York, v. 30, n. 1, p. 25-33, 1951.
AVRIL, J.L.; BRIFFORD, J.; BEINIS, J.P.; DUBRISAY, J. Etude de l'influence de l'antibiothérapie sur les résistances des entérobactéries de l'íntestin. Ann. Microbiol., Paris, v.131B, n.1, p.21-29, 1980.

BAMBURY, R.E. Synthetic antibacterial agents. In: BURGER, A., WOLFF, M., eds. Burger's Medicinal Chemistry. 4.ed., New York: Willey-Interscience, 1980. Part. 2, p.42-81.

BRYAN, G.T. In: BRYAN, G.T., ed. Carcinogenesis: A Comprehensive Survey. New York: Raven Press, 1978. p. $1-11$.

CARRON, M.; JULLIEN, M.A.; JULIA, M.T.; GARCZYNSKA, M. Propriétés antiseptiques de quelques nitro - 5 furfurilydène benzhydrazides. Etude du (nitro-5' furfurilydène-2) hydroxy-4benzhydrazide. Ann. Pharm. Fr., Paris, v.21, n.4, p.287-297, 1962 .

COLLINS, C.H.; LYNE, P.M.; GRANGE, J.M. Collins and Lyne's Microbiological Methods. 7.ed. Oxford: Butterworth-Heinemann, 1995. p. 493.

CRAIG, P.N. Interdependence between physical parameters and selection of substituent groups for correlation studies. J. Med. Chem., Columbus, v. 14, n. 8, p. 680684, 1971.

CROWCROFT, N.S. Methicillin-resistant Staphylococcus aureus and antimicrobial use in Belgian hospital. Infect. Control Hosp. Epidemiol., Thorofare, v.20, n. 1, p. 31-36, 1999.

DAYAN, J.; DEGUINGAND, C.; TRUZMAN, C. Study of the mutagenic activity of 6 hepatotoxic pharmaceutical drugs in the Salmonella typhimurium microsome test, and the HGPRT $\mathrm{Na}+/ \mathrm{K}+$ ATPase system in cultured mammalian cells. Mutat. Res., Amsterdam, v.157, n.1, p.1-12, 1985.

DESAI, D.; DESAI, N.; NIGHTINGALE, P.; ELLIOT, T.; NEUBERGER, J. Carriage of methicillin-resistant Staphylococcus aureus is associated with an increased risk of infection after liver transplantation. Liver Transpl., Los Angeles, v.9, n. 7, p. 754-759, 2003.

El-OBEID, H.A.; ELNIMA, E.I.; AL-BADR,A.A. Synthesis and antimicrobial activity of new furan derivatives. Pharm. Res., Stuttgart, v.1, p.42-43, 1985. 
FOYE, W.O.; LEMKE, T.L.; WILLIANS, D.A. Principles of medicinal chemistry. 4.ed. Baltimore: Williams \& Wilkins, 1995. p.995.

FUCHS, P.C.; KOOP, J., HAFNER, H.; KLEINER, U., PALLUA, N. MRSA - retrospective analysis of an outbreak in the burn centre Aachen. Burns, Wiltshire, v. 28, n. 6, p. $575-578,2002$.

FURLANETTO, M.; SANTOS, M.G.B.; TAVARES, L.C. Avaliação da influência de grupos substituintes sobre a atividade antimicrobiana de derivados 5-nitro-2tiofilidênicos. Rev. Bras. Cienc. Farm, São Paulo, v. 37, supl. 1, p. 62, 2001.

GAUDIO, A.C. Modelos clássicos de estudo quantitativo das relações entre estrutura-química e atividade biológica. Quim. Nova, São Paulo, v. 19, n. 3, p. 278-289, 1996.

GREEN, M.N. The effect of furacin (5-Nitro-2-Furaldehyde Semicarbazone) on the metabolism of bacteria. Arch. Biochem., New York, v. 19, n. 1, p. 397-405, 1948.

HAMMETT, L.P. The effect of structure upon the reactions of organic compounds. J. Am. Chem. Soc., Columbus, v. 59, n.1, p. 96-103, 1937.

HANSCH, C.; FUJTA, T. r-s-p- analysis: a method for the correlation of biological activity and chemical structure. J. Am. Chem. Soc., Columbus, v. 86, n.8, p. 1616-1626, 1964.

HANSCH, C.; LEO, A. Exploring QSAR: Fundamentals and Applications in Chemistry and Biology. Washington: American Chemical Society, 1995. 557p.

HANSCH, C.; LEO, A.; HOEKMAN, D. Exploring QSAR: hydrophobic, electronic, and steric constants. Washington: American Chemical Society, 1995. 348 p.

HIRANO, K.; YOSHINA, S.; OKAMURA, K.; SUZUKA, I. Electrophilic aspect of the antibacterial activity of nitrofuran derivatives. Bull. Chem. Soc. Jpn., Tokyo, v. 40, n. 10, p. 2229-2233, 1967.

HOPFINGER, A.J.; WANG, S.; TOKARSKI, J.S.; JIN, B.Q.; ALBUQUERQUE, M.; MADHAV,P.J.; DURAISWAMI, C. Construction of 3D-QSAR models using the 4D-QSAR analysis formalism. J.Am. Chem. Soc., Washington, v. 119, n. 43, p. 10509-10524, 1997.
KOROLKOVAS, A., BURCKHALTER, J. H. Quimica farmacêutica. Rio de Janeiro: Guanabara Dois, 1982. $783 \mathrm{p}$.

KOROLKOVAS, A. Essentials of medicinal chemistry, 2. ed., New York: John Wiley, 1988. p. 1204.

KUBINYI, H. QSAR: Hansch analysis and related approaches, New York: VCH, 1993. 240p.

LIEN, E.J. SAR: Side Effects and Drug Design. New York and Basel:Marcel Dekker Inc., 1987. p. 25-40.

LIZIOLI, A.; PRIVITERA, G.; ALLIATA, E.; BANFI, E.M.A., BOSELLI, L., PANCERI, M.L., PERNA, M.C., PORRETTA, A.D., SANTINI, M.G., CARRERI, V. Prevalence of nosocomial infections in Italy: result from the Lombardy survey in 2000. J. Hosp. Infect., Londres, v. 54, n. 2 , p. 141-148, 2003.

MASUNARI, A.; REZENDE, P.; TAVARES, L.C. Antimicrobial activity optimization of 5-nitro-2thiophylidene derivatives II. Eur. J. Pharm. Sci., São Paulo, v.13, p.S130 - S130, 2001. In: $3^{\text {rd }}$ Congress of Pharmaceutical Sciences, 2001, São Paulo.

MASUNARI, A.; TAVARES, L.C. Antimicrobial activity optimization of 5-nitro-2-thiophylidene derivatives against multi-resistant strains of Staphylococcus aureus. Drugs Fut., Barcelona, v.27. p.183 - 183, 2002. In: XVII ${ }^{\text {th }}$ International Symposium on Medicinal Chemistry, 2002, Barcelona.

MASUNARI, A.; NASCIMENTO, F.L.L.; FURLANETTO, M.; SANTOS, M.G.B.; PENNA, T.C.V.; MAMIZUKA, E.M.; TAVARES, L.C. Determinação da atividade antimicrobiana de derivados 5-nitro-2-tiofilidênicos frente às cepas padrão e multi-resistente de Staphylococcus aureus. Rev. Bras. Cienc. Farm, São Paulo, v. 39, supl.3, p. 192-194, 2003. In: VII Semana de Ciência e Tecnologia da FCF/USP, São Paulo.

MCDONALD, P.; MITCHELLI, E.; JOHNSON, H.; ROSSNEY, A.; HUMPHREYS, H.; GLYNN, G.; BURD, M.; DOYLE, D.; MCDONNELL, R. Epidemiology of MRSA: the North/South study of MRSA in Ireland 1999. J. Hosp. Infect., Londres, v. 54, n. 2, p. 130-134, 2003. 
OLIVEIRA, G.A.; DELL'AQUILA, A.M.; MASIERO, R.L.; LEVY, C.E.; GOMES, M.S.; CUI, L.; HIRAMATSU, K., MAMIZUKA, E.M. Isolation in Brazil of nosocomial Staphylococcus aureus with reduced susceptibility to vancomicin. Infect. Contr. Hosp. Epidem., Thorofare, v. 22, n. 7, p. 443-448, 2001.

PETRI JR., W.A. Antimicrobial Agents. In: HARDMAN, J.G., LIMBIRD, L.E., GILMAN, A.G. GOODMAN \& GILMAN'S: The pharmacological basis of therapeutics. 10 ed., New York: McGraw-Hill, 2001. p. 1171.

PISCOPO, E.; DIURNO, M.V.; GAGLIARDI, R.; MAZZANI, O., FRANCESCO, F.M., PARRILI, C. Structure-activity relationships of hidrazono derivatives of biological interest. Boll. - Chem. Soc. Ital. Biol. Sper., Napoli, v. 55, n. 4, p. 311-316, 1989.

REZENDE, P.; MASUNARI, A.; SANTOS, M. G. B.; MAMIZUKA, E. M.; VESSONI-PENNA, T. C.; TAVARES, L. C. Hansch Analysis of nifuroxazide analogues with antimicrobial activity against MRSA. Drugs Fut., Barcelona, v.27. p.192-192, 2002. In: XVII ${ }^{\text {th }}$ Symposium on Medicinal Chemistry, 2002, Barcelona.

SREIDER, C.M.; GRINBLAT, T.; STOPPANI, A.O.M. Catalysis of nitrofuran redox-cycling and superoxide anion production by heart lipoamide dehydrogenase. Biochem. Pharmacol., Oxford, v. 40, n. 8, p. 1849-1857, 1990.

SWAIN, C.G.; LUPTON Jr., E.C. Field and ressonance components of substituent effects. J. Am. Chem. Soc., Columbus, v. 90, n. 15, p. 4328-4337, 1968.

TAVARES, L.C. Relações quantitativas entre a estrutura química e a atividade antimicrobiana de análogos a nifuroxazida. São Paulo, 1993. 186p. (Tese de Doutorado - Faculdade de Ciências Farmacêuticas - Universidade de São Paulo).

TAVARES, L.C. Relações quantitativas estrutura-atividade em derivados 5-nitro-heterocíclicos com atividade antimicrobiana. São Paulo, 2003. 98p. [Tese de livre docência. Faculdade de Ciências Farmacêuticas, Universidade de São Paulo].
TAVARES, L.C.; AMARAL, A.T. Partition coefficient determination of nitrofurans derivatives for a QSAR study. In: WORKSHOP ON CHEMICAL STRUCTURE-BIOLOGICAL ACTIVITY, São Paulo, 1991. Resumos de Trabalhos. São Paulo: I.Q./USP. 1991. p. 8.

TAVARES, L.C.; CHISTÉ, J.J.; SANTOS, M.G.B.; PENNA, T.C.V. Synthesis and biological activity of nifuroxazide and analogs II. Boll. Chim. Farm., Milano, v. 138, n. 8, p. 432-436, 1999 a.

TAVARES, L.C.; MILANI, S.R.; RODRIGUES, A.M.; SANTOS, M.G.B.; PENNA, T.C.V. Síntese e determinação da atividade antimicrobiana de derivados do 5-nitro-2-furfurilideno. Rev. Farm. Quim., São Paulo, v.32, n.1, p.44-48, 1999 b.

TAVARES, L.C.; PENNA, T.C.V.; AMARAL, A.T. Synthesis and biological activity of nifuroxazide and analogs. Boll. Quim. Farm., Milano, v.136, n.3, p.244249, 1997.

TAVARES, L.C.; VESSONI-PENNA, T.C.; AMARAL, A.T. A QSAR study of nitrofurans derivatives with antimicrobial activity. In: WORKSHOP ON CHEMICAL STRUCTURE-BIOLOGICAL ACTIVITY, São Paulo, 1991. Resumos de Trabalhos. São Paulo: I.Q./USP. 1991.p. 2.

TAVARES, L.C. QSAR: A abordagem de Hansch. Quim. Nova, v. 27, n. 4, p. 631-639, 2004.

TOCHER, J.H. Reductive activation of nitroheterocyclic compounds. Gen. Pharmacol., Oxford, v. 28, n. 4, p. 485 487, 1997.

TOPLISS, J.G., Utilization of operational scheme for analog synthesis in drug design J. Med. Chem., Columbus, v. 15, n. 10, p. 1006-1010, 1972.

TORVALDSEN, S. The continuing evolution of methicillinresistant Staphylococcus aureus in western Australia. Infect. Contr. Hosp. Epidem., Thorofare, v. 20, p.133-135, 1999.

TUTE, M.S. History and objectives of quantitative drug design. In: HANSCH, C., SAMMES, G.P., TAYLOR, J.B. (eds) Comprehensive medicinal chemistry: The Rational Design, Mechanistic Study \& Therapeutic Application of Chemical Compounds. Oxford: Pergamon Press, 1990. v. 4, p. 1-31. 
ULMAR, T.M.; MITCHARD, M. The competitive inhibition of nitroredutase by some analogues of nitrofurantoin. Biochem. Pharmacol., New York, v.17, p.2057-2060, 1968.

VAN DE WATERBEEND, $\mathrm{H}$. The history of drug research: from Hansch to the present, QSAR, Weinheim, v. 11, n. 2 , p. 200-204, 1992.

VENUTI, M.C. The role of recombinant DNA technology in medicinal chemistry and discovery. In: WOLFF, M.E., ed. Burger's medicinal chemistry and drug discovery: principles and practice. 5 ed., New York: John Wiley \& Sons, 1995, v.1, p. 661-696.

VERLOOP, A. The sterimol approach to drug design. New York: Marcel Dekker, 1987. 112p.
VIODÉ, C.; BETTACHE, N.; CENAS, N.; KRAUTHSIEGEL, R.L.; CHAVIÉRE, G.; BALAKARA, N.; PÉRIE, J. Enzymatic reduction studies of nitroheterocycles. Biochem. Pharmacol., Oxford, v. 57, n. 5, p. 549-557, 1999.

VOSS, A.; MILATOVIC, D.; WALHAUCH-SCHWARZ, C.; ROSDHAL, V.T.; BRAVENY, I. Methicillin-resitant Staphylococcus aureus in Europe. Eur. J. Clin. Microbiol. Infect. Dis., Wiesbaden, v. 13, n. 1, p. 50-55, 1994.

VOZYAKOVA, T.I., OLEINIK, A.F., NOVITSKII, K.Y. Synthesis and reactions of arylfuran derivatives, V. Nauchn Konf. Khim. Teknol. Furanovykh Soedin (1978) 100-101: Chem. Abstr. 92 (1980) 180897.

Recebido para publicação em 15 de abril de 2005. Aceito para publicação em 09 de março de 2006. 